\title{
Centralized Gateway Concept for Precoded Multi-beam GEO Satellite Networks
}

\author{
Steven Kisseleff, Eva Lagunas*, Jevgenij Krivochiza, Jorge Querol, Nicola Maturo, \\ Liz Martinez Marrero, Juan Merlano-Duncan and Symeon Chatzinotas \\ Interdisciplinary Centre for Security, Reliability and Trust, University of Luxembourg, Luxembourg \\ *Corresponding Author: eva.lagunas@uni.lu
}

\begin{abstract}
Satellite Communications offer complementary benefits to terrestrial 5G/6G infrastructure, covering a wide range of use cases in need of ubiquitous coverage and reliability. However, to be as competitive as the terrestrial counterpart in terms of supplied throughput, satellite communications require a highly efficient use of the limited available spectrum. Linear precoding has demonstrated the ability to boost the spectral efficiency in the satellite domain, but raising a new issue: the bandwidth requirements of the feeder link. Deployment of several gateways, each of which precoding an independent cluster of beams causes performance degradation. Therefore, in this paper, we investigate the centralized gateway concept, where all digital baseband processes (including precoding) are implemented in a remote server connected via high speed fibers to the distributed remote gateways responsible for the downlink and uplink of the satellite radio frequency signals. In particular, we highlight the main technical challenges and provide a preliminary vision of potential solutions.
\end{abstract}

\section{INTRODUCTION}

Next-generation of geostationary satellite communication networks are expected to support extremely high data rates in order to maintain its competitiveness with respect to the technological advances of the terrestrial communications counterpart [1]. The challenge is to reduce the cost per bit, which can be effectively achieved with enhanced spectral efficiency. While the multibeam coverage of High Throughput Satellites (HTS) combined with the so-called linear precoding techniques have dramatically improved the satellite system throughput in the forward link [2], one of the main practical challenges still to be addressed is how to deal with the bandwidth shortage of the feeder link, which increases exponentially as it aggregates the traffic of all users. The feeder link is defined as the communication link between the gateway $(\mathrm{GW})$ and the satellite. Clearly, everything that is transmitted in the downlink (from satellite to Earth), has to be previously received by the satellite via the feeder link (from Earth to satellite). The latter represents a bottleneck for precoded systems, as the feeder link has typically limited capacity [3].

To overcome this bottleneck, the most popular methods involve the use of optical links [4], [5] and the GW switching [3], [6]. The former methods typically exploit the large bandwidth of optical signaling, which can accommodate a few Terabit/s while the latter is focused on exploiting the transmit diversity of multiple GWs via clever switching between them. However, the diversity alone is not sufficient to reach the required data rates, especially in the modern communication era [7]. As shown in [8], the multi-GW deployment not only relaxes the feeder link capacity constraint but can also achieve the best precoding performance if and only if there is one central coordinating entity in the network in charge of precoding all user signals. This have motivated the concept of jointly precoded transmissions over multiple GWs, which is currently being considered in the European Space Agency (ESA) project entitled "Prototype of a Centralized Broadband Gateway for Precoded Multi-beam Networks" [9].

Within a Centralized Gateway (CGW) concept, all digital baseband processes are implemented in multiple remote GWs connected via high speed fibers to the distributed remote gateways responsible for the downlink and uplink of the satellite radio frequency signals. On the path to the final design of this concept, various challenges need to be resolved. The most important ones can be summarized as follows:

- Synchronization aspects: To minimize the impact into the precoding performance, the signals transmitted via multiple GWs need to be accurately synchronized in time and frequency at the User Terminal (UT) receiver side.

- Precoding aspects: A centralized precoding involves joint precoding of high number of beams, resulting in increased computational complexity and latency. Furthermore, the synchronization errors may differ from one beam to another due to the multi-GW architecture and affect the Channel State Information (CSI).

- User scheduling aspects: The optimal joint precoding and user scheduling design is a non-convex and difficult to solve problem. However, a CGW architecture may increase the complexity affordability and allow to derive more advanced techniques considering user demandsatisfaction and fairness.

In this paper, we introduce the CGW concept and architecture, followed by a discussion on the main technical challenges and potential solutions.

The remainder of the paper is organized as follows. In Section II, the novel architectural concept for SatCom based on a centralized gateway is proposed and its expected features and benefits are discussed. Section III describes the technical challenges for the design and implementation of the proposed concept as well as suggested solutions to solve these problems. Subsequently, the paper is concluded in Section IV. 


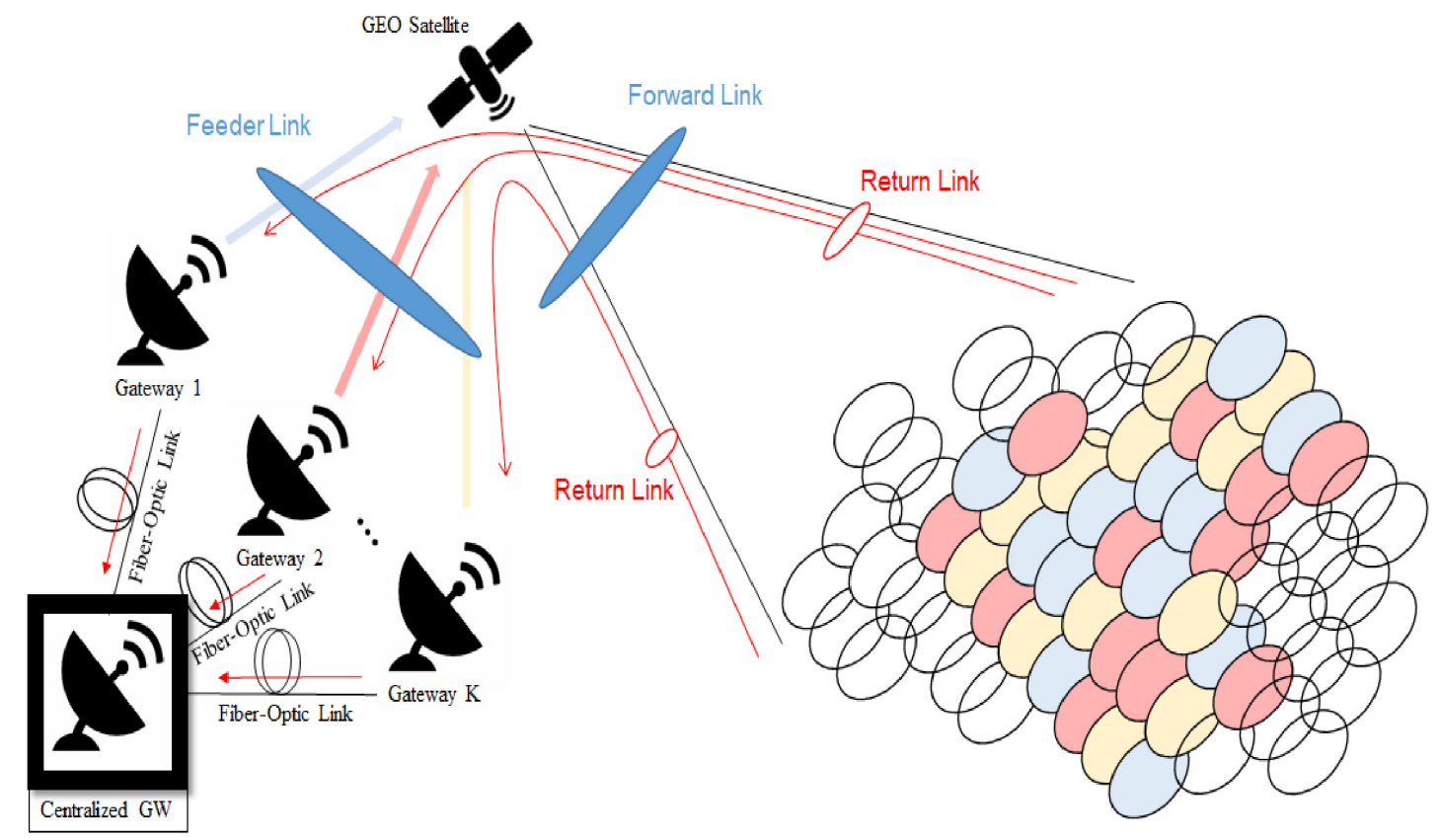

Fig. 1. Centralized GW concept for precoded multibeam GEO system. Multiple GWs are connected via fiber-optical links to a central unit, where joint signal processing for precoded feeder link transmissions and return link receptions take place.

\section{Centralized Gateway Concept}

The use of multiple gateways is a well-known solution in order to reuse the feeder link frequency resources through directive antennas [10]. The multi-GW deployment not only relaxes the feeder link capacity constraint but can also achieve the best precoding performance when there is one central coordinating entity in the network in charge of precoding all user signals. The concept of CGW involves a well-synchronized interconnected network of multiple GWs according to Fig. 1. Here, the CGW is responsible for all signal processing and higher layers operations, such as scheduling, precoding, synchronization, etc., while the remote GWs are only employed for the up-conversion of the signal, thus making them more similar to a simple Remote Radio Head (RRH) rather than a classical GW.

In the proposed architecture, all digital baseband processes are implemented in a remote server connected via high speed fiber optics to the distributed remote GWs responsible for the downlink and uplink of the satellite RF signals. The high speed optical fiber connections are especially crucial for the CGW concept, since the distances between distributed GWs can span hundreds and sometimes thousands of kilometers. Usually satellite operators have 2 options to achieve sufficient connectivity between their GW sites:

1) The satellite operators have a dedicated optical link that connect their GWs;

2) The satellite operators buy this service from a third part, usually a terrestrial operator.

The advantage of the first solution is that the satellite operator has its own dedicated link that can be managed independently. The main disadvantage is the very high cost of this dedicated connection. The second solution is much cheaper, but the satellite operator may have limited influence on the management of this part of the network. However, it has been shown in [11] that even with a limited signaling between multiple GWs, the precoding performance does not suffer too much degradation, such that the second option may not be too critical for the operator.

Since the complexity of the distributed GWs can be substantially reduced by moving the computational processes closer to the $\mathrm{CGW}$, the architecture shown in Fig. 1 is very similar to the Centralized or Cloud-based Radio Access Network (C-RAN), cf. [12]. Unlike in traditional RAN, where each access point is attached to a baseband unit (BBU) with limited computational power, in C-RAN, all calculations are carried out by a pool of BBUs, where joint processing (incl. precoding and pre-distortion, decoding, etc.) is applied. Through this, it is possible to account for computationally intensive tasks, which would not be possible to handle with a single BBU. The BBU pool may consist of thousands of BBUs implemented on the basis of FPGA and accelerated GPUs. The remaining parts of the access point remains a simple radio head with signal up- and down-conversion capabilities. Due to the promising features of C-RAN, it has been well studied for its application in $3 \mathrm{G}, 4 \mathrm{G}$ and $5 \mathrm{G}$. In order to be able to support the growing data traffic, number of users and services especially during rush hours, the computational capabilities of the BBU pool should be enough to cope with the peak demand. While the actual computation capabilities of C-RAN are not explicitly explained, the peak processing demands of the respective systems have been analyzed in [13], [14]. Correspondingly, this allows us to extrapolate the peak demands for the future 


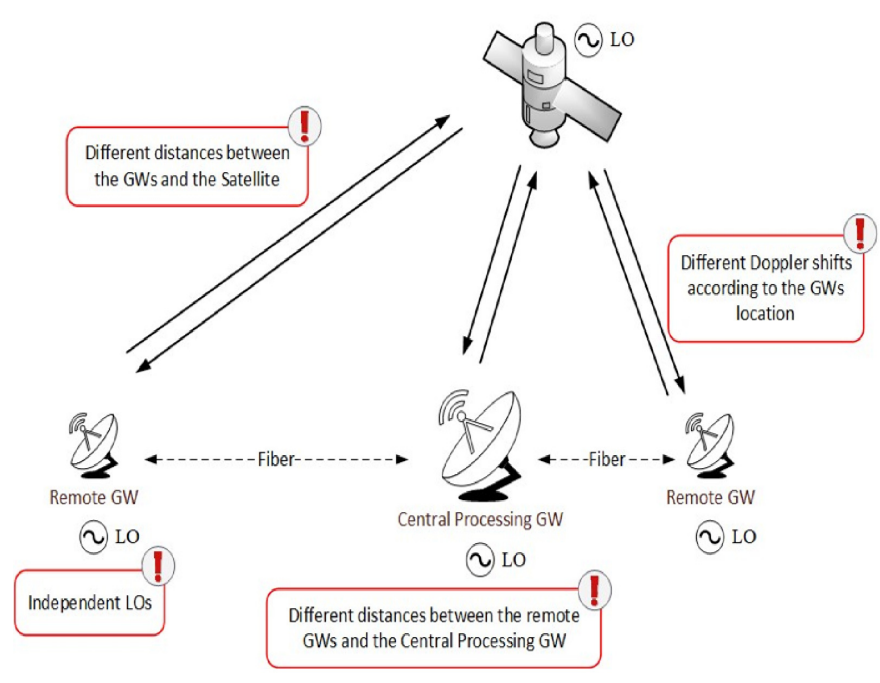

Fig. 2. Synchronization challenges of the CGW architecture.

systems to be in the order of several $10^{12}$ flops. Hence, future C-RAN supporting these peak demands should be able to have the corresponding capabilities.

Due to the envisioned integration of satellite and terrestrial communication systems, the CGW may need to cope with similar peak demands. Accordingly, the inner architecture of the CGW may be very similar to the C-RAN architecture comprising a pool of BBUs.

\section{TeChnical Challenges And Solutions}

\section{A. Synchronization Aspects}

In order to enable the joint signal processing at the CGW and jointly precoded transmissions from the distributed GWs, the communication between any remote GW and the CGW needs to be thoroughly synchronized. The two main synchronization aspects are time and frequency synchronization, which are detailed in the following subsections. Fig. 2 provides an overview of the major synchronization challenges of the CGW architecture.

1) Time synchronization: Typically, with a single GW, time misalignment can be only caused by different paths inside the payload [15]. However, in case of multiple GWs there, are two more sources of possible time misalignment between the transmitted signals:

- The difference in the distances between the central processing site and the peripheral GWs;

- The different distances between the peripheral GWs and the satellite itself.

Time misalignment has two different effects on the end-toend precoded communication chain; (i) impact on the CSI estimation since the Pilot Walsh-Hadamard sequences are not aligned, and (ii) degradation in the precoded signal combination seen by the receivers. Clearly, since the signal processing capabilities are available only in the central GW, it will be the central processing site to account for the time misalignment between the waveforms coming from the different remote GWs. Moreover, due to the different slant range, this problem is not dissimilar with respect to the problem discussed in [16]. Hence, pre-compensation based on an initial calibration-based measurements seems to be a viable solution in this case. In any case, an acceptable accuracy would be around 0.3 times the sampling time, which for the an oversampling factor of 4 , represents $-7.5 \%$ to $7.5 \%$ the symbol time.

2) Frequency synchronization: Frequency misalignment with a single GW typically do not pose any serious issues for the system design. However, in case of multiple GWs, even small GEO movements translate into problematic Doppler effects and thus frequency uncertainty. Specifically, multiple feeder links will be simultaneously transmitted from different remote GWs and therefore different Doppler effects can be expected according to their specific location. Since the system performance in terms of signal quality and packet error rate is typically very sensitive to differential phase variations among the channels, the frequency offsets need to be as small as possible thus putting extreme requirements on the synchronization accuracy. For this reason, Doppler pre-compensation techniques with the special focus on precoded systems need to be developed.

In addition to relatively small Doppler shifts due to the satellite motion, each GW employs its own local oscillator with a unique frequency offset and frequency drift. All these impairments need to be estimated and compensated in order to achieve the goal of sufficiently accurate joint precoding. This can be done using the technique described in [16]. The idea is to collect the channel state information (CSI) through the downlink in the remote GWs and then sent the estimated CSI back to the central GW that would apply precompensation on the generated signal in order to compensate the frequency misalignment. In order to improve the reliability of the estimated CSI by the remote GWs, a common reference signal can be used. Here, the simplest solution is to use the global positioning system (GPS) as common reference between the remote GWs.

\section{B. Precoding Aspects}

1) Complexity: The complexity of precoding comprises two components. The first component is the calculation of the precoder itself and depends on the type of employed precoder. Specifically, for linear precoding, this corresponds to the calculation of the precoder matrix. The second component is the precoding of a data stream using the designed precoder. For linear precoding, this corresponds to the multiplication of the precoder matrix with the input signal vector. These two components can be very computationally expensive for the CGW concept due to a large number of users to be jointly precoded.

The most popular precoder in satellite communication systems is the linear minimum mean-squared error (MMSE) precoder [17]. This precoder minimizes the error stemming from inter-beam interference as well as the ambient noise by employing a specifically designed regularization factor. A 
distinct advantage of this regularization is possibility to obtain a meaningful result even in case of ill-conditioned channel matrix. While the computation of the precoding matrix is mainly driven by the inversion of the channel matrix, this method remains still fairly low-complex.

There are many methods for obtaining the matrix inverse. The two most common ones are Gaussian elimination, Cholesky and QR decompositions. The selection of the decomposition algorithm strongly depends on the properties of the channel matrix. Although Gaussian elimination is universal for solving a linear equation, it is not efficient enough for cases for structured matrices, such as sparse matrices. The Cholesky decomposition and QR decomposition are two good and efficient alternatives. Complexity can be expressed in terms of floating point operations (flops) required to find the solution, expressed as a function of the problem dimension. The asymptotic complexity of Cholesky decomposition is $\frac{1}{3} N^{3}$ flops [18]. Although the asymptotic complexity of QR decomposition is known to be higher than Cholesky decomposition, the drawback of Cholesky is that it requires the channel matrix to be positive definite in order to be able to obtain a stable solution. whereas QR does not have this requirement. Hence, QR decomposition may be preferred in some cases. Below we provide an example of the corresponding operations per second required for computing the precoding matrix with the Cholesky method:

Input:
- $\mathrm{N}=200$ beams
- XFECFRAME length $=64800$ symbols (based on DVB-
S2X SF2)
- User bandwidth $=500 \mathrm{MHz}$ (Symbol Rate of 500
Msymbol $/ \mathrm{sec}$ )
Complexity of the Cholesky decomposition:
- $200^{3} / 3 / 64800=41.2$ operations/symbol
- $41.2 \cdot 500=20.6 \cdot 10^{9}$ operations/sec

The precoder calculation needs to be executed every time that the scheduled users changes (i.e. at PHY layer frame level) and even if the scheduled users are fixed, the precoder needs to be updated if there are variations in the channel conditions (the latter rarely occurs due to the large coherence time of GEO satellite channels). However, the precoding itself has to be executed in each symbol interval, thus making this operation typically more complex. The product of the precoding matrix with the symbols accounts for $N^{2}$ matrix-vector multiplications and this has to be done at each symbol (in the worst case).

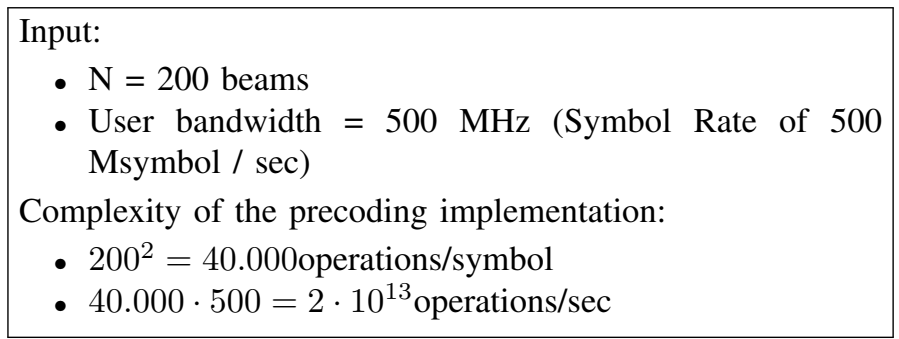

However, there are some practical limitations and some further complexity reduction techniques that can alleviate the previous complexity numbers.

2) Channel Nullification: During the channel estimation process each channel coefficient is estimated with determined uncertainty. In addition, when the interference received power is very low, it might be unfeasible to detect and estimate the co-channel coefficient. When this happens, a null replaces the channel coefficient that is not able to be detected. The latter is known as channel nullification.

Based on [19] and Table C.6 of the same document, the detectability threshold is defined using the carrier-overinterference ratio $C / I \geq 15 \mathrm{~dB}$. However, the actual threshold depends on the time synchronicity of the interferering signals. Assuming perfect time synchronization, the interference-overnoise ratio $I / N$ may become more relevant, as it does not take into account the relative power of the interference with respect to the main signal [20].

The nullification of the channel matrix occurs, if the required $C / I$ during the pilot transmission for channel estimation is low, i.e. below $20 \mathrm{~dB}$. Then, the receiver is not able to accurately estimate the channel coefficients of the interfering beams and assumes that they are zero (nullified). This effect leads to performance degradation for the precoder, since interference is not properly minimized. This degradation manifests often performance degradation in terms of per-beamrate. To avoid this, the $C / I$ value needs to be increased, e.g. using precoding.

In addition, Walsh-Hadamard (WH) sequences-based channel estimation (which is typically employed) is limited by at most 32 orthogonal sequences. Correspondingly, at most 32 channel coefficients can be estimated using unique WH sequences. With respect to the ideal channel matrix estimation (unlimited number of orthogonal sequences), this effect can be viewed as a nullification and has exactly the same impact on the performance. The hope is however that only the strongest 32 channel coefficients will be determined, which would at least reduce the impact of nullification as much as possible.

A positive effect from the nullification is related to the computational complexity. In some cases, up to $70 \%$ of the elements of channel matrix are assumed to be zero. This can be easily observed in Fig. 3, where the number of CSI components that can be measured are shown for different $C / I$ thresholds (denoted by $\delta$ ). Hence, the number of multiplications for the precoding can be substantially reduced leading to complexity savings. 


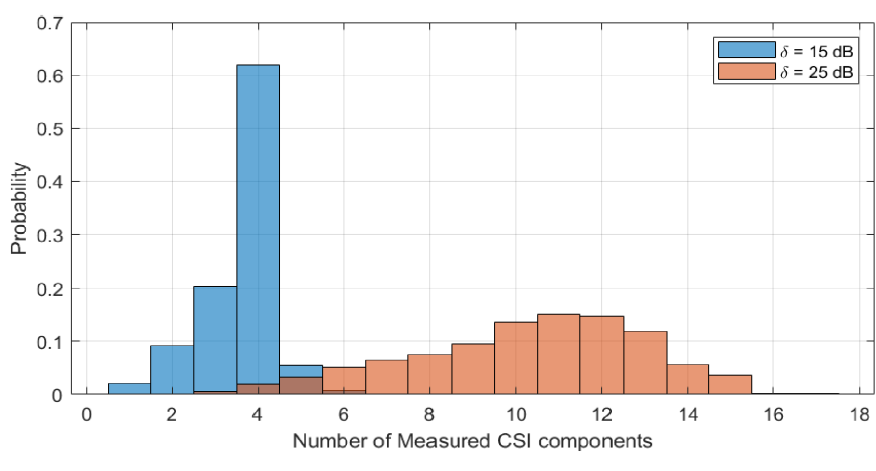

Fig. 3. Number of CSI components that can be measured assuming different $\mathrm{C} / \mathrm{I}$ thresholds (denoted by $\delta$ )

TABLE I

MEAN AND STARDARd DEVIATION OF THE CHANNEL ESTIMATE (REFERENCE SIGNAL SNR=10 DB) [19]

\begin{tabular}{|c|c|c|c|c|c|c|}
\hline & \multicolumn{7}{|c|}{ C/I [dB] } \\
\hline & & 0 & 4 & 8 & 12 & 16 \\
\hline \multirow{2}{*}{ Amplitude [dB] } & Mean & 0.08 & 0.10 & 0.69 & 1.94 & Not Detectable \\
& Std & 0.12 & 0.26 & 0.37 & 0.58 & Not Detectable \\
\hline \multirow{2}{*}{ Phase [deg] } & Mean & 0.30 & 0.36 & 1.10 & 4.62 & Not Detectable \\
& Std & 0.94 & 1.50 & 2.41 & 5.37 & Not Detectable \\
\hline
\end{tabular}

3) Imperfect CSI: As mentioned, depending on the percarrier $C / I$, each channel coefficient is estimated with a certain uncertainty. In [19], the uncertainty is modeled with an additive Gaussian error on the mean and phase components with the parameters shown in Table I.

Furthermore, there is a delay between the time for which the channel coefficients are estimated and the time frame for which the precoder matrix based on those channels is applied. During this delay, the channel might change due to different effects, such as differential phase noise (due to different oscillators at remote GWs) and user mobility (causing channel alteration in terms e.g. satellite antenna phase and Doppler shift). Even with a frequency synchronization algorithm in place, the remaining phase noise artifacts among the GWs are hard to remove and need to be taken into account as an additional Gaussian-like noise. The second effect can be very harmful for the precoding performance. However, the motion of the users with respect to the GEO satellite with a stationary geographical location is typically rather low, such that this effect is mitigated.

\section{Scheduling Aspects}

The analysis of precoding performance is usually done by assuming an independent upper layer which selects users at each time instance. However, it has been shown that user scheduling has a significant impact on the system performance.

Joint user scheduling and precoding design is a coupled problem, because both aspects are closely related and heavily impact each other's performance. The general scheduling challenges have been discussed in [21] and are summarized below:
- Multicast or Frame-based Scheduling: the symbols of one frame, regardless of the information they convey, are delivered to multiple users. These users need to receive the entire frame and to be able to decode it in order to extract the information dedicated to them. For this, the modulation and coding scheme (MODCOD) utilized in the frame should be selected according to the needs of the user with the worst channel conditions among all users scheduled to receive this frame. This requirement typically leads to significant performance losses unless the channels of the multicast users scheduled are as close as possible in order to avoid strong discrepancies in the multicasted users' channel conditions.

- Channel Orthogonality: To minimize the impact of inter-beam interference, channel orthogonality between multicast groups (i.e. users scheduled across beams) need to be as orthogonal as possible [22].

Since the CSI is obtained by the CGW via return link and therefore, with higher complexity affordability, the optimization of the user scheduling can be achieved by employing more advanced methods [2]. In general, the grouping of the users within the same beam is a non-trivial task and might require a thorough optimization, which also takes into account the potentially heterogeneous user demands, cf. [24].

1) Imperfect/outdated CSI: As discussed, scheduling decisions are sometimes taken based on the CSI feedback coming from the user terminals. Another possibility is to perform precoding/scheduling without any CSI feedback, but rather resorting to a CSI estimation at the $\mathrm{GW}$ side based on the location information of the users. Such strategy would allow to reduce considerably the signalling and could potentially enable precoding over a DVB-S2 air interface. To account for the CSI uncertainty and resulting power losses due to channel mismatch, a proper margin needs to be incorporated in the MODCOD allocation.

2) Quality-of-Service and User Fairness: The scheduling is also challenged by the need to deliver multimedia services in broadband multibeam satellite system. The nature of the packet traffic in broadband services is bursty, i.e. the data rate needed to support the different services is not constant. As a consequence, the conventional forward link satellite schedulers need to be adapted to optimize bandwidth (capacity) utilization not only based on the propagation conditions but also based on the Quality of Service (QoS), in the presence of traffic flows generated by services with different requirements (e.g. latencysensitive versus speed-hunger traffic). Furthermore, most of the designs have focused on maximization of throughput, which is known to be an unfair solution from the individual user perspective. Each user has a minimum service requirement that the operator is supposed to provide, and which needs to be considered in the scheduling design [22], [24].

\section{CONCLUSIONS AND REMARKS}

In this paper, a novel concept of a Centralized GW has been introduced. We propose to move all processing tasks to a central unit, thus leaving only the functionality of remote radio 
head to the original GWs. This concept enables more advanced precoding methods in order to increase the throughput of the feeder link, which is expected to become the bottleneck of future satellite communication systems. Furthermore, joint precoding of all beams will provide a better demand satisfaction compared to the individual precoding of clustered beams associated with the respective GWs. Regarding the feasibility of this concept, we investigated the technological challenges related to synchronization, precoding and scheduling, and proposed potential solutions to these challenges.

\section{ACKNOWLEDGMENT}

This work has been supported by the European Space Agency (ESA) funded activity CGD: Prototype of a Centralized Broadband Gateway for Precoded Multi-beam Networks. Please note that the views of the authors of this paper do not necessarily reflect the views of ESA. The author would like to thank J. Grotz and S. Andrenacci from SES, Luxembourg, for their helpful advice on various practical and technical aspects of this work.

\section{REFERENCES}

[1] O. Kodheli et al., "Satellite Communications in the New Space Era: A Survey and Future Challenges," IEEE Communications Surveys Tutorials, vol. 23, no. 1, pp. 70-109, 2021.

[2] M. A. Vazquez, A. Perez-Neira, D. Christopoulos, S. Chatzinotas, B. Ottersten, P. Arapoglou, A. Ginesi, and G. Taricco, "Precoding in Multibeam Satellite Communications: Present and Future Challenges,' IEEE Wireless Communications, vol. 23, no. 6, pp. 88-95, December 2016.

[3] A. Gharanjik, B. S. M. R. Rao, P.-D. Arapoglou, and B. Ottersten, "Large scale transmit diversity in Q/V band feeder link with multiple gateways," in IEEE 24th Annual International Symposium on Personal, Indoor, and Mobile Radio Communications (PIMRC), 2013, pp. 766-770.

[4] S. Poulenard, M. Crosnier, and A. Rissons, "Ground segment design for broadband geostationary satellite with optical feeder link," Journal of Optical Communications and Networking, vol. 7, no. 4, pp. 325-336, 2015.

[5] R. Saathof, R. den Breeje, W. Klop, S. Kuiper, N. Doelman, F. Pettazzi, A. Vosteen, N. Truyens, W. Crowcombe, J. Human et al., "Optical technologies for terabit/s-throughput feeder link," in 2017 IEEE International Conference on Space Optical Systems and Applications (ICSOS), 2017, pp. 123-129.

[6] A. Gharanjik, B. S. M. R. Rao, P.-D. Arapoglou, and B. Ottersten, "Gateway Switching in Q/V Band Satellite Feeder Links," IEEE Communications Letters, vol. 17, no. 7, pp. 1384-1387, 2013.

[7] S. K. Sharma, S. Chatzinotas, and P.-D. Arapoglou, Satellite communications in the $5 G$ era. Institution of Engineering \& Technology, 2018.

[8] G. Zheng, S. Chatzinotas, and B. Ottersten, "Multi-gateway cooperation in multibeam satellite systems," in 2012 IEEE 23rd International Symposium on Personal, Indoor and Mobile Radio Communications-(PIMRC). IEEE, 2012, pp. 1360-1364.
[9] ESA CGD Project: Prototype of a Centralized Broadband Gateway for Precoded Multi-beam Networks. [Online]. Available: https: //wwwfr.uni.lu/snt/research/sigcom/projects/esa_cgd

[10] V. Joroughi, M. Vazquez, and A. Perez-Neira, "Multiple Gateway Precoding with Per Feed Power Constraints for Multibeam Satellite Systems," in European Wireless Conference, 2014, pp. 1-7.

[11] C. Mosquera, R. López-Valcarce, T. Ramírez, and V. Joroughi, "Distributed precoding systems in multi-gateway multibeam satellites: Regularization and coarse beamforming," IEEE Transactions on Wireless Communications, vol. 17, no. 10, pp. 6389-6403, 2018.

[12] J. Wu, Z. Zhang, Y. Hong, and Y. Wen, "Cloud radio access network (c-ran): a primer," IEEE network, vol. 29, no. 1, pp. 35-41, 2015.

[13] Y. Liao, L. Song, Y. Li, and Y. A. Zhang, "How much computing capability is enough to run a cloud radio access network?" IEEE Communications Letters, vol. 21, no. 1, pp. 104-107, 2016.

[14] I. Gomez, V. Marojevic, and A. Gelonch, "Resource management for software-defined radio clouds," IEEE micro, vol. 32, no. 1, pp. 44-53, 2011.

[15] S. Andrenacci, S. Chatzinotas, A. Vanelli-Coralli, S. Cioni, A. Ginesi, and B. Ottersten, "Exploiting Orthogonality in DVB-S2X through Timing Pre-compensation," in Advanced Satellite Multimedia Systems Conference and Signal Processing for Space Communications Workshop (ASMS/SPSC), 2016, pp. 1-8.

[16] L. Martinez Marrero, J. C. Merlano Duncan, J. Querol, S. Chatzinotas, A. J. Camps Carmona, and B. Ottersten, "Effects of multiple oscillator phase noise in precoding performance," in International Communications Satellite Systems Conference (ICSSC), 2019.

[17] D. Christopoulos, S. Chatzinotas, G. Zheng, J. Grotz, and B. Ottersten, "Linear and nonlinear techniques for multibeam joint processing in satellite communications," EURASIP journal on wireless communications and networking, vol. 2012, no. 1, pp. 1-13, 2012.

[18] R. Hunger, Floating point operations in matrix-vector calculus. Munich University of Technology, Inst. for Circuit Theory and Signal Processing, 2005.

[19] "Digital Video Broadcasting (DVB); Second generation framing structure, channel coding and modulation systems for Broadcasting, Interactive Services, News Gathering and other broadband satellite applications; Part 2: DVB-S2 Extensions (DVB-S2X)," https://www.etsi.org/deliver/etsi_tr/102300_102399/10237602/01.01. 01 60/tr 10237602v010101p.pdf, accessed: 2021-06.

[20] ESA OPTIMUS Project: COptimized transmission techniques for satcom unicast interactive traffic . [Online]. Available: https://wwwfr.uni.lu/snt/research/sigcom/projects/optimus_optimized_ transmission_techniques_for_satcom_unicast_interactive_traffic

[21] S. Kisseleff, E. Lagunas, T. S. Abdu, S. Chatzinotas, and B. Ottersten, "Radio resource management techniques for multibeam satellite systems," IEEE Communications Letters, 2020.

[22] E. Lagunas, S. Andrenacci, S. Chatzinotas, and B. Ottersten, "Crosslayer forward packet scheduling for emerging precoded broadband multibeam satellite system," in Advanced Satellite Multimedia Systems Conference (ASMS), Sep. 2018, pp. 1-8.

[23] P. Honnaiah, E. Lagunas, D. Spano, N. Maturo, and S. Chatzinotas, "Demand-Based Scheduling for Precoded Multibeam High-Throughput Satellite Systems," in IEEE Wireless Communications and Networking Conference (WCNC), 2021, pp. 1-6. 